\title{
PROPOSING A MULTI-CRITERIA PATH OPTIMIZATION METHOD IN ORDER TO PROVIDE A UBIQUITOUS PEDESTRIAN WAYFINDING SERVICE
}

\author{
Mohammadreza Sahelgozin ${ }^{\mathrm{a}, *}$, Abolghasem Sadeghi-Niaraki ${ }^{\mathrm{b}}$, Shokouh Dareshiri ${ }^{\mathrm{c}}$ \\ GIS Dept., Geoinformation Technology Center of Excellence, Faculty of Geodesy\&Geomatics Engineering \\ K.N.Toosi University of Technology, Tehran, Iran \\ a,c (sahelgozin,dareshiri)@mail.kntu.ac.ir@b a.sadeghi@kntu.ac.ir
}

KEY WORDS: Multi-Criteria, Pedestrian Wayfinding, Ubiquitous Service, Context-Aware

\begin{abstract}
:
A myriad of novel applications have emerged nowadays for different types of navigation systems. One of their most frequent applications is Wayfinding. Since there are significant differences between the nature of the pedestrian wayfinding problems and of those of the vehicles, navigation services which are designed for vehicles are not appropriate for pedestrian wayfinding purposes. In addition, diversity in environmental conditions of the users and in their preferences affects the process of pedestrian wayfinding with mobile devices. Therefore, a method is necessary that performs an intelligent pedestrian routing with regard to this diversity. This intelligence can be achieved by the help of a Ubiquitous service that is adapted to the Contexts. Such a service possesses both the Context-Awareness and the User-Awareness capabilities. These capabilities are the main features of the ubiquitous services that make them flexible in response to any user in any situation. In this paper, it is attempted to propose a multi-criteria path optimization method that provides a Ubiquitous Pedestrian Way Finding Service (UPWFS). The proposed method considers four criteria that are summarized in Length, Safety, Difficulty and Attraction of the path. A conceptual framework is proposed to show the influencing factors that have effects on the criteria. Then, a mathematical model is developed on which the proposed path optimization method is based. Finally, data of a local district in Tehran is chosen as the case study in order to evaluate performance of the proposed method in real situations. Results of the study shows that the proposed method was successful to understand effects of the contexts in the wayfinding procedure. This demonstrates efficiency of the proposed method in providing a ubiquitous pedestrian wayfinding service.
\end{abstract}

\section{INTRODUCTION}

Navigation systems help the users of different transportation modes to get to their destinations effectively. Many novel applications for navigation systems have emerged, including navigation of vehicles, pedestrians or wheelchair users. An important application of such systems is Wayfinding. It is a key function of Location Based Services (LBS) (Zheng et al., 2013). Most of the previous studies in wayfinding methods were for the vehicles. However, vehicle navigation systems are not applicable for pedestrians because there are a number of differences between the navigation of vehicles and pedestrians. These differences have some reasons. Pedestrians usually have higher degrees of freedom and they are not bounded to the road network. Passing through squares or parks that are inaccessible for cars is possible for pedestrians. Moreover, pedestrians need higher precisions than the GPS systems which are used in the cars for positioning. They are also more heterogeneous in terms of movement than car drivers (Holone et al., 2007; Millonig and Schechtner, 2007).

A significant number of studies were performed about pedestrian wayfinding. However, they had some limitations. For example, some studies concentrated on only one criterion such as length, safety (Furukawa and Nakamura, 2013) or health (Sharker et al., 2012) to find the best route. Some other researches, took into account more than one criterion simultaneously; however, majority of them focused on multi-criteria path finding for vehicles (Niaraki and Kim, 2009; Pahlavani et al., 2012; Pahlavani and Delavar, 2014), or they developed multi-criteria wayfinding methods generally without specifying for pedestrians (Nadi and Delavar, 2011; Mohammadi and Hunter, 2012).
Apart from that, the most important limitation of the previous attempts in pedestrian wayfinding is the lack of proper consideration to service flexibility. They are not attentive to the diversity in environmental conditions of the users and their preferences. In fact, their criteria are constant in all situations. Nevertheless, the importance of such a flexibility in pedestrian wayfinding is significant. Because, whether a certain route is a more appropriate choice than another or not, strongly depends on situational preferences and also on user preferences (Niaraki and Kim, 2009). In other words, navigation with mobile devices is affected by the variability of contexts and the diversity of users' preferences and capabilities (Delikostidis et al., 2015).

Therefore, a method is needed to perform an intelligent routing with regard to the differences in the contexts and in the users. This intelligence can be achieved by developing a Ubiquitous Service. Such a service is adapted to the contexts and to its users so that it is able to serve the right information in the right situation to the right person (Zipf and Jöst, 2006). In fact, it understands both its environment and its users which leads it to be called as Context-Aware. Context can be interpreted as the information that are used to characterize the situation of an entity (Biancalana et al., 2013); Therefore, context-awareness means being aware of the physical environment that surrounds a user and their mobile device (Schmidt et al., 1999). In the recent years, some researches focused on context-aware pedestrian navigation systems. For example, Ludwig et al. (2014) considered the influence of the contexts on the salience of a landmark that is suggested by the system. Nevertheless, there has not been a significant attempt in the previous studies to propose a multi-criteria path optimization method for pedestrian wayfinding purposes that provides a ubiquitous service.

\footnotetext{
* Corresponding author
} 
In this paper, it is attempted to propose a multi-criteria path optimization method in order to provide a Ubiquitous Pedestrian Way Finding Service (UPWFS); that possesses both ContextAwareness and User-Awareness capabilities. To achieve this goal, at first, UPWFS is defined in a proposed conceptual framework in Section 2. With regard to the constructed conceptual framework, UPWFS is then modelled mathematically as a multi-criteria decision making problem in Section 3. Then in Section 4, the proposed pedestrian wayfinding method is described. In order to evaluate the performance of the proposed method, an experiment was carried out on the data of a local district in Tehran whose results and discussions are represented in Section 5. Finally, a conclusion of the study is provided in Section 6.

\section{CONCEPTUALIZATION}

Before modelling the problem quantitatively, a conceptualization of UPWFS is provided in this section by proposing a framework. UPWFS has two main elements: Context and User, also the latter consists of two sub-elements that are User Characteristics and User Preferences. Each of these elements is introduced by a number of factors. These factors influence the appropriateness of a path and thus, they have effects on wayfinding of a pedestrian. Reviewing the literature, influences of a number of factors are highlighted in the researches. Factors that are confirmed more by the previous studies are extracted and categorized in Table 1. It should be noted that the objectives of the mentioned studies in the table are not necessarily the same as of the current study; however, they believed in the importance of the mentioned factors in some similar problems.

In this study, the fact that whether a path is appropriate with regard to the context and user or not is expressed by four criteria: Length, Safety, Difficulty and Attraction of the path. These criteria are affected by the factors introduced in Table 1 . The influence of each factor on these four criteria are represented in the proposed framework (Figure 1).

in the framework, the criterion Safety is divided in two parts: Accident Safety and Social Safety; the former is the safety of pedestrians in terms of the fact that how much they are probable to have an accident with vehicles while crossing across the streets; and the latter is the security of the pedestrians in terms of the probability of becoming disturbed or attacked by an offender. These two parts are not homogeneous and they are not affected by the same factors. That is why, Kim et al. (2011) or Florez et al. (2014) did also separate these two parts of the criterion Safety in their studies.

For example, age of a pedestrian is a factor that has effect on both accident safety and social safety; thus in the framework, there are two arrows from the factor Age to both parts of the criterion Safety. However, the factor Gender may not affect the probability of accident very obviously and only social safety may be impressed by the gender of a user; hence, only one arrow from the factor Gender to the criterion Safety can be seen in the framework.

Furthermore, it is important to say that Land Use distribution is a specification of the path; however, it does not determine attraction of the path on its own. In fact, attractiveness of a path is also defined with regard to the interests and preferences of each user. In this study, the element User Preferences describes a user's preferred land use distribution on the way.

After conceptualizing the problem, it is needed to apply a model to describe the problem mathematically. In the next section, it is attempted to represent a mathematical model of the proposed framework.

Table 1. UPWFS elements and its effective factors

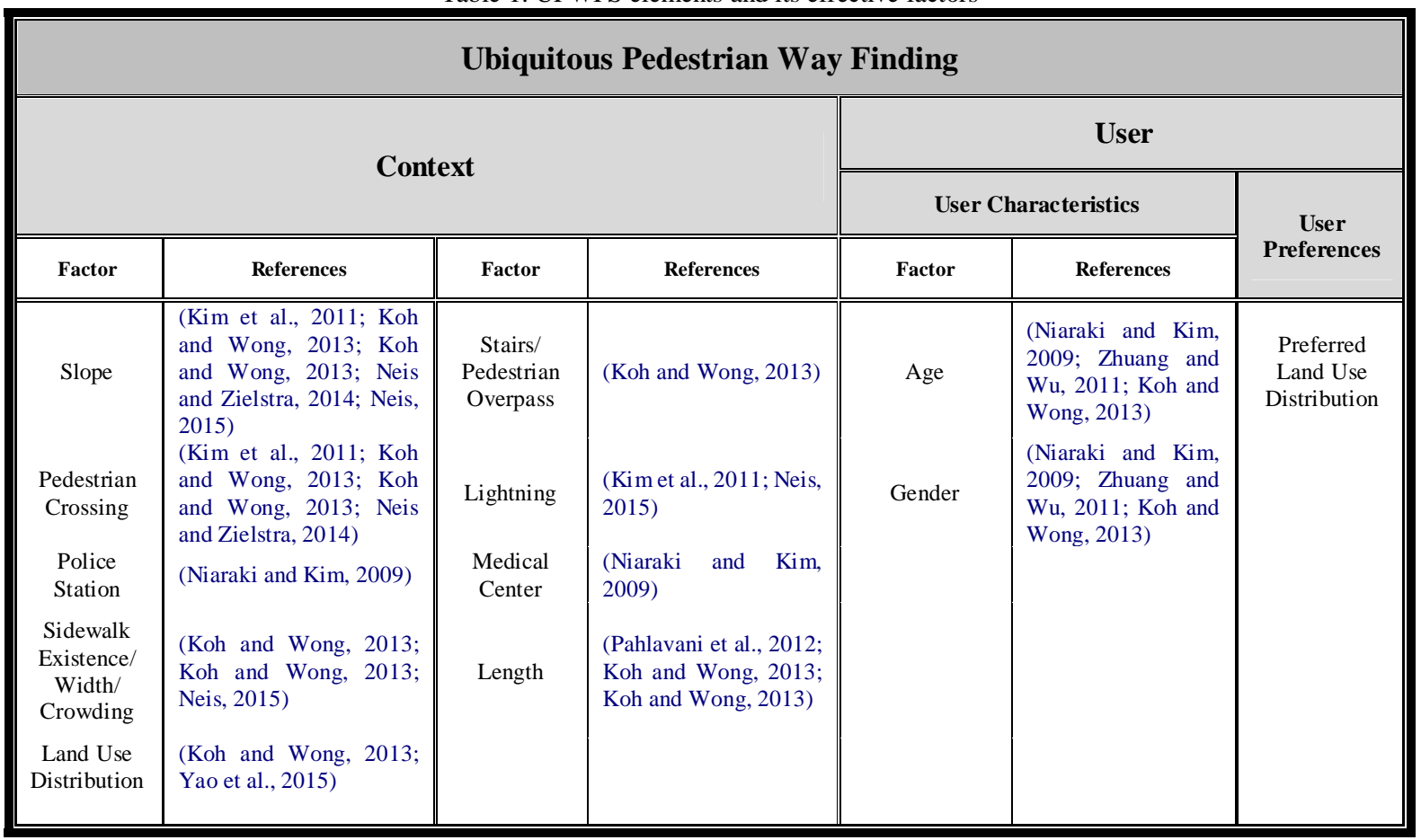




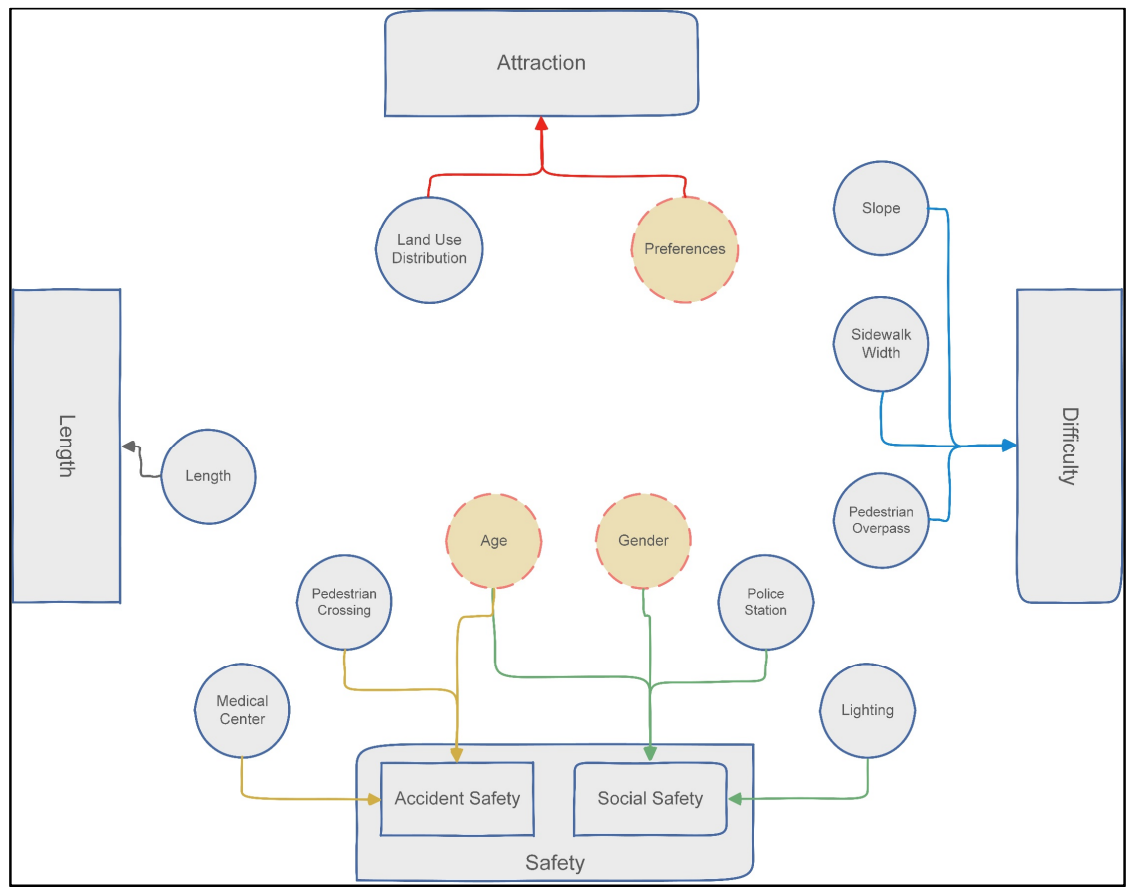

Figure 1. The proposed Framework for UPWFS

\section{MATHEMATICAL MODELLING}

In this section, the objective is to model UPWFS mathematically as a multi-criteria decision making problem. In order to accomplish this, for each criterion, corresponding measurement function for pedestrian network segments is formulated based on its own effective factors. Then impedance of a segment is determined according to these functions.

As the first criterion, Length of a segment (Length $h_{\text {seg }}$ ) is calculated by normalization of its length with regard to the minimum and maximum boundaries. However, in order to measure safety of a segment, more calculations are needed. As it was mentioned before, the criterion Safety is divided in two parts: Accident Safety and Social Safety. Each part is measured separately since the factors influencing them are heterogeneous. Accident Safety for each segment (AccidentSafety seg $_{\text {) is }}$ calculated by its influencing factors as below:

$$
\begin{aligned}
& \text { AccidentSafety }_{\text {seg }}= \\
& \quad w_{C} * \text { Crossing }_{\text {seg }}+w_{M C} * \text { MedicalCenter }_{\text {seg }}
\end{aligned}
$$

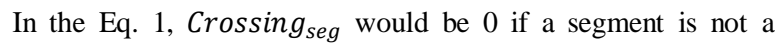
pedestrian crossing; otherwise, it would be 1. Also, MedicalCenter $_{\text {seg }}$ is existence of any medical centers in a segment which is either 0 or 1 . In addition, the value for $w_{C}$ and $w_{M C}$ are obtained by Analytic Hierarchy Process (AHP) (Saaty, 1990) (Table 2).

Similar to the Accident Safety, Social Safety of a segment (SocialSafety $_{\text {seg }}$ ) is calculated by Eq. 2 .

$$
\begin{aligned}
& \text { SocialSafety }_{\text {seg }}= \\
& \quad w_{L} * \text { Lighting }_{\text {seg }}+w_{P S} * \text { PoliceStation }_{\text {seg }}
\end{aligned}
$$

It should be noted that in this study, the factors Age and Gender are not considered in the calculation of a segment's safety in order to simplify the task. Instead, they are considered when the aggregate impedance function for the segment is constructed in Eq. 5.

Table 2. Extracted weights of the factors by AHP method

\begin{tabular}{|c|c|c|}
\hline Notation & Factor & Weight \\
\hline \multicolumn{3}{|c|}{ Accident Safety } \\
\hline$w_{C}$ & Crossing & 0.9 \\
\hline$w_{M C}$ & Medical Canter & 0.1 \\
\hline \multicolumn{3}{|c|}{ Social Safety } \\
\hline$w_{L}$ & Lighting \\
\hline$w_{P S}$ & Police Station & 0.8 \\
\hline \multicolumn{3}{|c|}{ Difficulty } \\
\hline$w_{S}$ & Slope \\
\hline$w_{S W}$ & Sidewalk Width & 0.449 \\
\hline$w_{P O}$ & Pedestrian Overpass & 0.169 \\
\hline
\end{tabular}

For measuring difficulty of a segment, influencing factors are summarized in Slope of the path, Sidewalk Width and Pedestrian Overpass. Thus Difficulty of a segment (Difficulty seg $_{\text {) }}$ ) is measured as the same as Eq. 3 .

$$
\begin{gathered}
\text { Difficulty }_{\text {seg }}=w_{S} * \text { Slope }_{\text {seg }}+ \\
w_{S W} *\left(1-\text { SidewalkWidth }_{\text {seg }}\right)+ \\
w_{P O} * \text { PedestrianOverpass } \\
\text { seg }
\end{gathered}
$$

It has been described that to consider influencing factors of safety and difficulty of a segment, they should be normalized with regard to their lower and upper boundaries. However, this approach is not feasible for those factors that influence the criterion Attraction; because they are related to a user's personality that has no meaningful boundaries. In this study, land use distribution of the path is considered as the influencing factor on its attraction. The amount of similarity between land use distribution of a segment and what the active user actually interests in is obtained by applying Vector Space Model (VSM). To apply VSM, for each segment a vector is defined whose 
elements are specified to each class of land use. If a specific land use exists in the segment, corresponding element in the vector would be set to 1 ; otherwise 0 . Another similar vector is defined for the user ranks to each class of land use. If an active user likes a class, the corresponding element in the vector would be set to 1 and if not, it would be set to 0. Finally, using VSM, similarity of the segment's land use distribution and the user's interesting land use distribution is calculated by measuring the cosine similarity between the two vectors (Eq. 4).

$$
\text { Cosine Similarity }=\frac{v_{s} \cdot v_{u}}{\left|v_{s}\right|\left|v_{u}\right|}
$$

Where $v_{s}$ and $v_{u}$ are vector of the segment and vector of the user, respectively.

Finally, to calculate a segment's impedance that considers all the above criteria, constructed functions are aggregated by weighted summation in Eq. 5. AHP is again used to weigh criteria by consideration to experts' pairwise comparisons of the criteria. Furthermore, in this step, age and gender of the user is considered in the weighting process. In other words, weights are flexible with the changes in the age and gender of a user. In Table 3, a classification of the age and gender is represented. For each class, priorities of the criteria are provided. With regard to these priorities, AHP is performed to obtain suitable weights for each age and gender group of users.

$$
\begin{aligned}
& \text { Impedance }_{\text {Seg }}=w_{\text {Len }} * \text { Length }+ \\
& w_{\text {ASaf }} * \text { AccidentSafety }+ \\
& w_{\text {SSaf }} * \text { SocialSafety }+w_{\text {Dif }} * \text { Difficulty }+ \\
& w_{\text {Att }} * \text { Attraction }
\end{aligned}
$$

Table 3. Classification of the age and gender of users

\begin{tabular}{|c|c|c|}
\hline Age & Gender & $\begin{array}{c}\text { Priorities of the } \\
\text { Criteria }\end{array}$ \\
\hline $0-12$ & Male & (L AS SS D A) \\
\hline $0-12$ & Female & (L AS SS D A) \\
\hline $12-18$ & Male & (L SS A AS D) \\
\hline $12-18$ & Female & (L SS A AS D) \\
\hline $18-60$ & Male & (L A AS SS D) \\
\hline $18-60$ & Female & (L A SS AS D) \\
\hline $60+$ & Male & (L D AS A SS) \\
\hline $60+$ & Female & (L D AS A SS) \\
\hline
\end{tabular}

\section{PROPOSED METHOD FOR UBIQUITOUS PEDESTRIAN WAYFINDING}

In this section, a multi-criteria path optimization method is proposed based on the model described in Section 3. The proposed method provides a ubiquitous pedestrian wayfinding service that is flexible according to the contexts and users. This service is able to suggest the shortest, the safest, the easiest and the most attractive route among a large number of possible routes from a pedestrian's origin to his/her destination.

A general overview of what happens step-by-step in the proposed method is represented in Figure 2. The procedure is summarized in five steps. At first, when an origin and a destination is defined for an active user, values for the variables of the contexts and the user are set. Then, by the help of these values and the mathematical functions developed in Eq.s 1-4, value of each criterion for each segment is calculated in the second step. It should be noted that the criteria values are not constant for a specific segment; conversely, it changes flexibly as the contexts or the user changes.

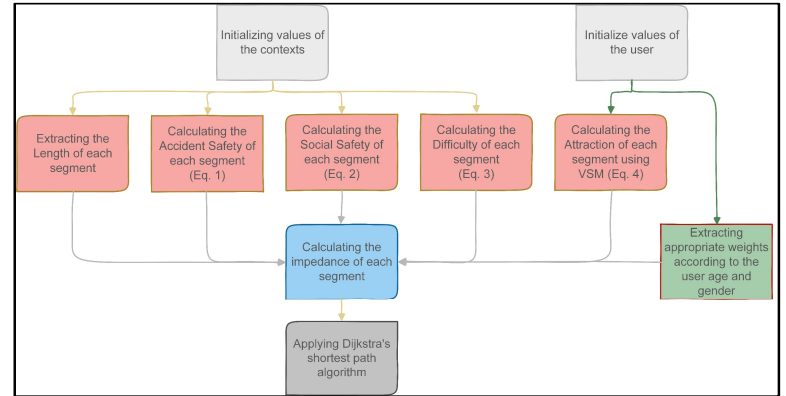

Figure 2. Steps of the proposed ubiquitous pedestrian wayfinding method

In the third step, in order to aggregate these functions in a unique value, AHP weights are extracted according to the age and gender of the user (Table 3). Then, by the help of the extracted weights, the impedance of each segment is calculated and stored in a Cost Matrix. Finally, by applying the Dijkstra's algorithm, the best path that satisfies all the criteria is derived. This route is going to be suggested to the active user as the shortest, the safest, the easiest and the most attractive path between his/her current location and the destination.

\section{EVALUATION OF THE PROPOSED METHOD}

To provide an opportunity for evaluating performance of the proposed wayfinding method in the real situations, data of a local district in Tehran was considered as the case study. The area was chosen in a way that all specifications of the pedestrian network such as existence of sidewalks, width of sidewalks, land use distribution, existence of medical centres or existence of police stations were diverse in the area.

In this study, the pedestrian network was constructed so that it would be able to respond to the needs of the model introduced in Section 3. Its segments are coinciding on centrelines of the sidewalks, pedestrian crossings and pedestrian overpasses. The constructed network, parcels' land uses and other necessary spatial features were gathered in ArcMap 10.1 with the help of the official Tehran GIS data and a complementary field survey. All features of the segments such as sidewalk widths, are defined as attributes in the geo-dataset. Also, the proposed method was implemented in Matlab 2013 environment as an application. This application gets values of the contexts variables and the user variables as inputs in order to extract and visualize the best path.

The experiment had two phases. In the first phase, a number of experimental cases were designed. In each case, age, gender and interests of the active user were changed. Figure 3 shows two specific cases. In this figure, two different routes were suggested by the application in each case from a specific node to another. One was suggested to a male user who is 40 years old and prefers residential areas (Figure 3-a); and the other was suggested to a 25 -year-old female for whom open areas (parks and urban green spaces) are interesting (Figure 3-b).

Although the origin and the destination were the same in the cases, the suggested route by the proposed method was changed. This has two reasons. First, changes in age and gender of the user amongst the two cases resulted in a change in priorities of the criteria (Table 3 ) and consequently caused a change in weights of the criteria in the segment impedance function (Eq. 5). Second, the users' interesting land use vectors were different; thus, Attraction values of the segments were not the same in the two cases. Suggesting disparate routes by the application in each case, 
demonstrates the flexibility of the proposed method in response to the different users.

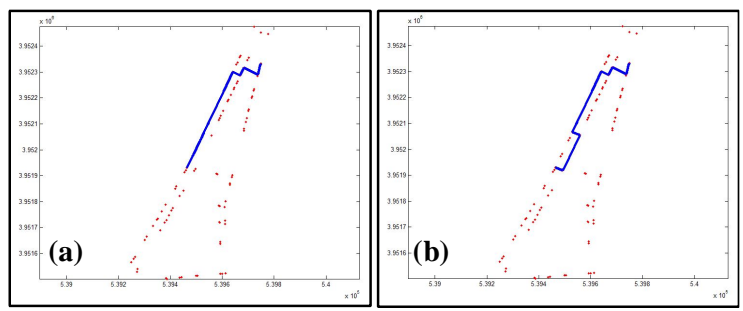

Figure 3. Comparison of the best route for two experimental cases: (a) Male-40-Residential (b) Female-25-Open Areas

Second phase of the experiment was assessment of the suggested route by the proposed method in comparison to the best feasible routes in terms of each criterion separately. Again, the experimental case for the 40-year-old male was considered and the path that is suggested for him by the application (Figure 4-a) was compared with the shortest route (Figure 4-b), safest route according to the Accident Safety (Figure 4-c), safest route according to the Social Safety (Figure 4-d), easiest route (Figure 4-e) and the most attractive route (Figure 4-f).

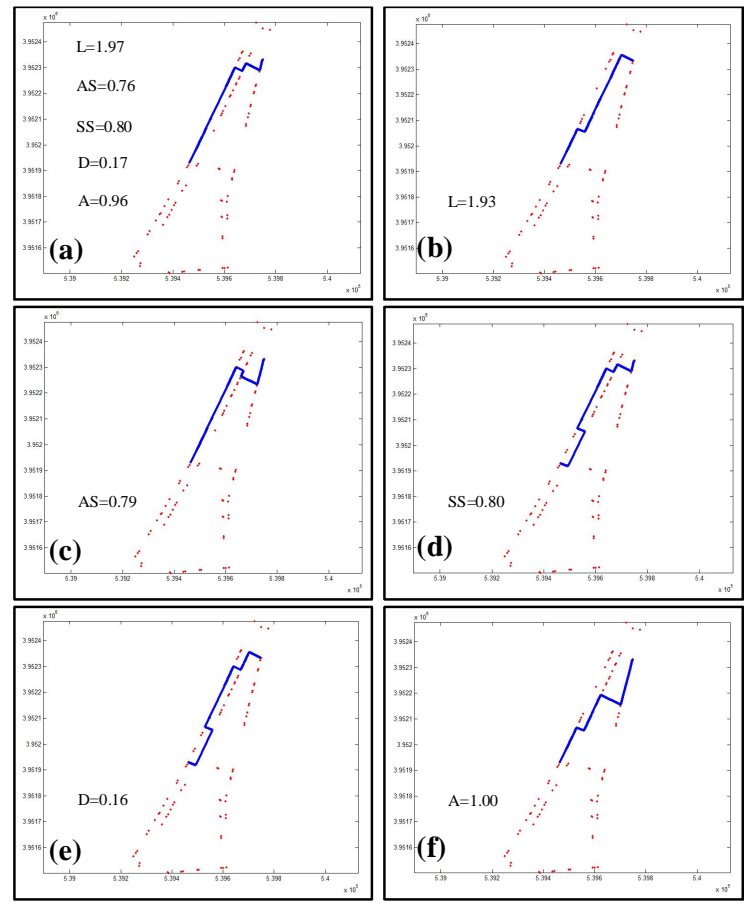

Figure 4. Suggested route by the proposed method (a) in comparison to the best routes in terms of length (b), accident safety (c), social safety (d), difficulty (e) and attraction (f)

As can be seen in the figures above, if we consider only one particular criterion separately, a best route in terms of that criterion exists that have a better measure for the considered criterion in comparison to the suggested route by the application. For example, the suggested route (Figure 4-a) was longer than the shortest feasible route, it had equal or lower safety measures than the safest routes (accident and social), it was more difficult to walk compared to the easiest route and finally it had less attraction than the most attractive route. However, in the singlecriterion modes, other criteria got worse values to allow the considered criterion to become the best. Therefore, although the suggested route by the application seems to be less appropriate in terms of each criterion separately, the combination of all the criteria was averagely improved in this route. In other words, all the criteria were taken into account simultaneously in the route finding procedure to suggest a route that is concurrently the shortest, the safest, the easiest and the most attractive choice.

\section{CONCLUSION}

In this study, a multi-criteria path optimization method was proposed in order to provide a Ubiquitous Pedestrian Way Finding Service (UPWFS). Context-Awareness and UserAwareness were the main features of UPWFS that made it possible for the wayfinding service to be flexible in response to any user in any situation. Commonly, pedestrians choose the shortest path from an origin to a destination; however, individual or environmental situations may make the user choose another route that satisfies other criteria. Therefore, apart from ubiquity, the proposed wayfinding method was also multi-criteria. Criteria of the proposed method were summarized in Length, Safety, Difficulty and Attraction of the route.

By reviewing results of the proposed method evaluation, it can be concluded that the proposed method was successful to understand effects of the contexts in wayfinding. In other words, when a user changed, the method applied characteristics and preferences of the new user in order to suggest a more appropriate route for $\mathrm{him} / \mathrm{her}$. Therefore, the proposed method is able to improve ubiquity of the wayfinding services for pedestrians. In addition, the proposed method suggested routes that were not necessarily the shortest routes. In fact, it seeks a path that satisfies all the Length, Safety, Difficulty and Attraction criteria simultaneously. Thus, the proposed method was also successful to be multi-criteria.

However, some limitations remained with the method. At first, the considered factors in the framework may not be the all; for example, in the developed model, contexts are limited to the constant specification of the route such as slope. On the other hand, adding new factors in the model may result in some inaccuracies. Thus, an arrangement is needed for adding further variable contexts such as time or weather conditions in the model as a future work. In addition, such a method needs special pedestrian network that is not available in previous datasets. By overcoming these limitations, the proposed method would be the basis of ubiquitous pedestrian services which make walking more attractive. This will encourages people to walk more and results in decreases in fuel consumptions and weather pollutions.

\section{REFERENCES}

Biancalana, C., Gasparetti, F., Micarelli, A. and Sansonetti, G., 2013. An approach to social recommendation for context-aware mobile services. ACM Transactions on Intelligent Systems and Technology (TIST), 4(1), pp. 1:1-1:32.

Delikostidis, I., van Elzakker, C. P. and Kraak, M.-J., 2015. Overcoming challenges in developing more usable pedestrian navigation systems. Cartography and Geographic Information Science,(ahead-of-print), pp. 1-18.

Florez, J., Muniz, J. and Portugal, L., 2014. Pedestrian Quality of Service: Lessons from Maracanã Stadium. Procedia-Social and Behavioral Sciences, 160, pp. 130-139. 
Furukawa, H. and Nakamura, Y., 2013. A Pedestrian Navigation Method for User's Safe and Easy Wayfinding. Human-Computer Interaction. Users and Contexts of Use, Springer: 156-165.

Holone, H., Misund, G. and Holmstedt, H., 2007. Users are doing it for themselves: Pedestrian navigation with user generated content. In: Next Generation Mobile Applications, Services and Technologies, NGMAST'07. The 2007 International Conference on, IEEE.

Kim, S., Joo, Y. and Park, S., 2011. Pedestrian Path Findings using Multi-Factors Affected Walking. In: Proceedings of the Geospatial World Forum.

Koh, P. and Wong, Y., 2013. Influence of infrastructural compatibility factors on walking and cycling route choices. Journal of Environmental Psychology, 36, pp. 202-213.

Koh, P. P. and Wong, Y. D., 2013. Comparing pedestrians' needs and behaviours in different land use environments. Journal of Transport Geography, 26, pp. 43-50.

Ludwig, B., Müller, M. and Ohm, C., 2014. Empirical Evidence for Context-aware Interfaces to Pedestrian Navigation Systems. KI-Künstliche Intelligenz, 28(4), pp. 271-281.

Millonig, A. and Schechtner, K., 2007. Developing landmarkbased pedestrian-navigation systems. Intelligent Transportation Systems, IEEE Transactions on, 8(1), pp. 43-49.

Mohammadi, E. and Hunter, A., 2012. Multi-Criteria Path Finding. ISPRS-International Archives of the Photogrammetry, Remote Sensing and Spatial Information Sciences, 1, pp. 157159.

Nadi, S. and Delavar, M. R., 2011. Multi-criteria, personalized route planning using quantifier-guided ordered weighted averaging operators. International Journal of Applied Earth Observation and Geoinformation, 13(3), pp. 322-335.

Neis, P., 2015. Measuring the reliability of wheelchair user route planning based on volunteered geographic information. Transactions in GIS, 19(2), pp. 188-201.

Neis, P. and Zielstra, D., 2014. Generation of a tailored routing network for disabled people based on collaboratively collected geodata. Applied Geography, 47, pp. 70-77.

Niaraki, A. S. and Kim, K., 2009. Ontology based personalized route planning system using a multi-criteria decision making approach. Expert Systems with Applications, 36(2), pp. 22502259.

Pahlavani, P. and Delavar, M. R., 2014. Multi-criteria route planning based on a driver's preferences in multi-criteria route selection. Transportation research part C: emerging technologies, 40, pp. 14-35.

Pahlavani, P., Delavar, M. R. and Frank, A. U., 2012. Using a modified invasive weed optimization algorithm for a personalized urban multi-criteria path optimization problem. International Journal of Applied Earth Observation and Geoinformation, 18, pp. 313-328.

Saaty, T. L., 1990. How to make a decision: the analytic hierarchy process. European journal of operational research, 48(1), pp. 9-26.
Schmidt, A., Beigl, M. and Gellersen, H.-W., 1999. There is more to context than location. Computers \& Graphics, 23(6), pp. 893901.

Sharker, M. H., Karimi, H. A. and Zgibor, J. C., 2012. Healthoptimal routing in pedestrian navigation services. In: Proceedings of the First ACM SIGSPATIAL International Workshop on Use of GIS in Public Health, ACM.

Yao, S., Loo, B. P. and Lam, W. W., 2015. Measures of activitybased pedestrian exposure to the risk of vehicle-pedestrian collisions: Space-time path vs. potential path tree methods. Accident Analysis \& Prevention, 75, pp. 320-332.

Zheng, J., Zhang, Z., Ciepluch, B., Winstanley, A. C., Mooney, P. and Jacob, R., 2013. A PostGIS-based pedestrian way finding module using OpenStreetMap data. In: Geoinformatics (GEOINFORMATICS), 2013 21st International Conference on, IEEE.

Zhuang, X. and Wu, C., 2011. Pedestrians' crossing behaviors and safety at unmarked roadway in China. Accident Analysis \& Prevention, 43(6), pp. 1927-1936.

Zipf, A. and Jöst, M., 2006. Implementing adaptive mobile GI services based on ontologies: Examples from pedestrian navigation support. Computers, Environment and Urban Systems, 30(6), pp. 784-798. 